\title{
Sorting nexin 24 is required for $\alpha$-granule biogenesis and cargo delivery in megakaryocytes
}

Joanne Lacey, ${ }^{1}$ Simon J. Webster, ${ }^{1}$ Paul R. Heath, ${ }^{2}$ Chris J. Hill, ${ }^{3}$ Lucinda Nicholson-Goult, ${ }^{4}$ Bart E. Wagner, ${ }^{4}$ Abdullah O. Khan, ${ }^{5}$ Neil V. Morgan, ${ }^{5}$ Michael Makris ${ }^{1}$ and Martina E. Daly ${ }^{1}$

1Department of Infection, Immunity and Cardiovascular Disease, University of Sheffield, Sheffield; ${ }^{2}$ Sheffield Institute for Translational Neuroscience (SITraN), Department of Neuroscience, University of Sheffield, Sheffield; ${ }^{3}$ Department of Molecular Biology and Biotechnology, University of Sheffield, Sheffield; ${ }^{4}$ Histopathology Department, Royal Hallamshire Hospital, Sheffield and 5 Institute of Cardiovascular Sciences, College of Medical and Dental Sciences, University of Birmingham, Birmingham, UK.

\author{
Correspondence: \\ Martina E. Daly \\ M.Daly@sheffield.ac.uk \\ Received: $\quad$ July 14,2021 \\ Accepted: January 3, 2022. \\ Prepublished: January 13, 2022. \\ https://doi.org/10.3324/haematol.2021.279636 \\ (02022 Ferrata Storti Foundation \\ Haematologica material is published under \\ a CC-BY-NC license (c) (1) \&
}

\begin{abstract}
Germline defects affecting the DNA-binding domain of the transcription factor FLI1 are associated with a bleeding disorder that is characterized by the presence of large, fused $\alpha$-granules in platelets. We investigated whether the genes showing abnormal expression in FLI1-deficient platelets could be involved in platelet $\alpha$-granule biogenesis by undertaking transcriptome analysis of control platelets and platelets harboring a DNA-binding variant of FLI1. Our analysis identified 2,276 transcripts that were differentially expressed in FLI1-deficient platelets. Functional annotation clustering of the coding transcripts revealed significant enrichment for gene annotations relating to protein transport, and identified Sorting nexin 24 (SNX24) as a candidate for further investigation. Using an induced pluripotent stem cell-derived megakaryocyte model, SNX24 expression was found to be increased during the early stages of megakaryocyte differentiation and downregulated during proplatelet formation, indicating tight regulatory control during megakaryopoiesis. CRISPR-Cas9 mediated knockout (KO) of SNX24 led to decreased expression of immature megakaryocyte markers, CD41 and CD61, and increased expression of the mature megakaryocyte marker CD42b $(P=0.0001)$, without affecting megakaryocyte polyploidisation, or proplatelet formation. Electron microscopic analysis revealed an increase in empty membrane-bound organelles in SNX24 KO megakaryocytes, a reduction in $\alpha$-granules and an absence of immature and mature multivesicular bodies, consistent with a defect in the intermediate stage of $\alpha$-granule maturation. Co-localization studies showed that SNX24 associates with each compartment of $\alpha$-granule maturation. Reduced expression of CD62P and VWF was observed in SNX24 KO megakaryocytes. We conclude that SNX24 is required for $\alpha$-granule biogenesis and intracellular trafficking of $\alpha$-granule cargo within megakaryocytes.
\end{abstract}

\section{Introduction}

The ETS transcription factor Friend leukemia virus integration 1, or FLI1, plays a fundamental role in megakaryopoiesis by cooperating with the ETS factor GA binding protein, alpha subunit (GABPA), to regulate the expression of multiple megakaryocyte-specific genes expressed during the early and late stages of megakaryopoiesis., ${ }^{1,2}$ Partial deletion of chromosome 11, in a region that includes the gene encoding FLI1 (11q23.3-24), is associated with Paris Trousseau syndrome, which is characterized by dysmegakaryopoiesis in the bone marrow. ${ }^{3,4}$ Affected individuals have an increased tendency to bleed, as well as thrombocytopenia characterized by the presence of large platelets containing large fused $\alpha$-granules in the circulation. ${ }^{5-7}$

Germline defects in FLI1 have also been described. ${ }^{8-10} \mathrm{In}-$ deed, we previously reported an enrichment of heterozygous FL/1 defects among patients with excessive bleeding in association with a significant reduction in $\delta$-granule secretion, and mild thrombocytopenia. ${ }^{8}$ Further characterization of two FL/1 alterations predicting amino acid substitutions in the ETS DNA-binding domain of FLI1 showed that they disrupted transcriptional activity and would therefore cause a reduction in the expression of megakaryocyte-specific genes, providing an explanation for the bleeding tendency observed in the patients. ${ }^{8}$ The identification of a homozygous FL/1 defect, which predicted a substitution in the ETS domain and resulted in a defect in transcription among members of a family affected by a bleed- 
ing disorder that resembled Paris Trousseau syndrome and was characterized by thrombocytopenia and the presence of abnormally large $\alpha$-granules in a subpopulation of platelets, suggested that the abnormal platelet granules were due to loss of FLI1 activity. ${ }^{9}$ The identification of two further FL/1 defects affecting residues in the ETS domain of FLI1 which were associated with the presence of giant $\alpha$-granules and depletion of $\delta$-granules supports this hypothesis. ${ }^{10}$

While the role of platelet degranulation in maintaining vascular integrity has long been recogniszd, the pathways leading to the assembly and exocytosis of platelet $\alpha$ - and $\delta$-granules, and the genes that regulate them, remain to be fully established. The biogenesis of $\alpha$ - and $\delta$-granules shares some common features, but the pathways involve distinct protein trafficking machinery. ${ }^{11,12}$ Both granules are derived through a process of budding from the trans-Golgi network to form vesicles that merge with early endosomes and mature into multivesicular bodies. ${ }^{13-15}$ The $\alpha$-granules can also be derived from the platelet membrane through clathrin-coated pit-mediated endocytosis to form vesicles that traffic to the early endosomes. 15,16 Studies in patients with inherited granule storage disorders, ${ }^{17-19}$ combined with extensive analyses of platelet lysates, and studies in mice carrying mutations in different secretion genes, have allowed characterization of the cargo of platelet storage granules, and provided essential information on the secretory machinery of platelets. ${ }^{20-22}$

The abnormally large $\alpha$-granules and reduced number of $\delta$ granules, and the defect in $\delta$-granule secretion that have been described in platelets from patients harboring FL/1 defects likely reflect the disruptions in gene expression that occur either directly or indirectly as a result of the abnormal transcriptional activity of FLI1. ${ }^{8-10}$ Furthermore, some of the genes showing disrupted expression in FLI1-deficient platelets displaying granule abnormalities could potentially be involved in platelet granule biogenesis under physiological conditions. We explored this hypothesis by undertaking transcriptome analysis of platelets from subjects harboring a deleterious defect in FLI1 (c.1028A>G; p.Tyr343Cys) to identify differentially expressed genes encoding proteins which may be involved in platelet granule biogenesis and secretion, and identified a role for Sorting nexin 24 (SNX24) in platelet granule biogenesis using wild-type and SNX24 knockout (KO) induced pluripotent stem cell (iPSC)-derived megakaryocytes to model maturation and platelet production.

\section{Methods}

\section{Subjects and platelet transcriptome analysis}

Total platelet RNA was isolated from $50 \mathrm{~mL}$ samples of peripheral blood from two heterozygous carriers of the c.1028A>G transition in FL/1 and from three sex-matched healthy individuals. All subjects were studied in parallel on two separate occasions. The study was approved by the $\mathrm{Na}-$ tional Research Ethics Service Committee (REC reference: 06/MRE07/36).

Following differential centrifugation to obtain platelet-rich plasma, total platelet RNA was isolated using Trizol (see Online Supplementary Methods). Two hundred nanograms of RNA (RIN >7 as measured on the Agilent Bioanalyzer) were converted to double-stranded cDNA and transcriptome analysis was carried out using human Clariom D Assay chips (Thermo Fisher), which were washed and stained according to the manufacture's standard protocols. The arrays were scanned using the Affymetrix $30007 \mathrm{G}$ scanner, and the .CEL files analyzed using Transcriptome Analysis Console (TAC) 4.0 software (Thermo Fisher) to identify genes showing $>2$ and $<-2$ fold log change and $P<0.05$.

Functional annotation clustering was carried out for those genes which were differentially expressed using the Database for Annotation, Visualization and Integrated Discovery (DAVID; http://david.abcc.ncifcrf.gov/) with the default settings and a low stringency setting. ${ }^{23,24}$

\section{Induced pluripotent stem cell differentiation to megakaryocytes}

The Gibco episomal human iPSC line was cultivated feederfree on Geltrex-coated flasks and maintained in StemFlex medium (Thermo Scientific). iPSCs were differentiated to mature megakaryocytes and proplatelets as described previously. ${ }^{25,26}$ Details of the cell culture conditions and differentiation of iPSC are included in the Online Supplementary Methods.

\section{Generation of the SNX24 knockout cell line}

The SNX24 KO cell line was generated using the Alt-R RNP system (Integrated DNA Technologies; IDT). SNX24 crRNA and Atto-555 labeled tracrRNA were annealed and the complex incubated with HiFi Cas9 V3 (IDT) to form stable RNP complexes, which were introduced into iPSC using Lipofectamine Stem (Life Technologies). For single-cell cloning, StemFlex medium was supplemented with CloneR (Stemcell Technologies) and the manufacturer's workflow was followed (see Online Supplementary Methods for further details).

\section{Gene expression analysis of SNX24 knockout induced pluripotent stem cells}

Gene expression of lineage-specific and cellular markers was measured during SNX24 KO iPSC differentiation by quantitative polymerase chain reaction (qPCR) using an ABI 7900 HT analyzer (Applied Biosystems). Further details are included in the Online Supplementary Methods, and all oligonucleotide sequences are listed in Online Supplementary Table S1.

\section{Characterization of megakaryocytes derived from SNX24 knockout induced pluripotent stem cells} Differentiation of SNX24 KO cells to proplatelet-forming 
megakaryocytes was assessed by immunofluorescent staining of cells, and imaging using a Zeiss A1 confocal microscope. Further details of the antibodies used, and the methodology are provided in the Online Supplementary Methods.

\section{Transmission electron microscopy of platelets and megakaryocytes}

FLI1-deficient platelets and iPSC-derived megakaryocytes were examined by transmission electron microscopy (TEM) using a Philips 400 and a FEI Tecnai transmission electron microscope, respectively. Further details are provided in the Online Supplementary Methods.

\section{Statistical analysis}

Results are expressed as means \pm standard deviation. Unless otherwise specified, statistical significance was determined using a Student $t$-test with $P<0.05$ considered to be statistically significant. Analyses were performed using GraphPad Prism software.

\section{Results}

\section{Transcriptome analysis of FLI1-deficient platelets}

Transcriptome analysis was undertaken using Human Clariom $D$ Assay chips for platelets from a father (P1) and son (P2), both of whom were heterozygous for the c.1028A>G FL11 variant predicting a p.Tyr343Cys substitution in the DNA-binding domain of FLI1. Both subjects were recruited to the UK GAPP study with a history of excessive bleeding and a suspected inherited platelet disorder, which was characterized by mild thrombocytopenia and a profound reduction in platelet ATP secretion in response to thrombin. The clinical features, and genotypic and phenotypic characteristics of both subjects have been reported previously. ${ }^{8}$ Electron microscopic examination of platelets from both P1 and P2 revealed the presence of giant and fused $\alpha$-granules similar to those previously described in platelets from subjects with FL11 defects (Figure 1A).,9,10 Platelet transcriptomes were also analyzed from three healthy male subjects ( $\mathrm{C} 1, \mathrm{C} 2$ and $\mathrm{C} 3$ ). Transcripts were considered to be significantly differentially expressed if they had a $>2$ or $<-2$ fold log change, and a $P<0.05$. Hierarchical clustering and principal component analysis showed clear differences between the FLI1-deficient platelets from P1 and P2 and normal platelets from C1, C2 and C3 (Figure 1B and Online Supplementary Figure S1A). Comparison of gene expression in platelets from the two cases with the c.1028A>G FL/1 variant with that in normal platelets ( $\mathrm{C} 1, \mathrm{C} 2$ and $\mathrm{C} 3$ ) identified 2,276 significantly differentially expressed transcripts (926 downregulated and 1350 upregulated) in the FLI1-deficient platelets (Figure $1 \mathrm{C}$ and Online Supplementary Tables S2 and S3). The 30 coding transcripts displaying the greatest up- and downregulation in expression in FLI1-deficient platelets relative to normal platelets are indicated in Figure 1D, while Online Supplementary Tables $S 4$ and $S 5$ list all coding transcripts showing significant down- and up-regulation in FLI1-deficient platelets.

To identify genes for further investigation, functional annotation clustering of the 1,487 differentially expressed coding transcripts was undertaken using the DAVID tool (Online Supplementary Table S6). This identified 234 clusters, which were enriched for multiple classes of annotation categories. Given our interest in platelet granule biogenesis and secretion, we focused on the gene ontology (GO) terms associated within the top four clusters: protein transport, cell-cell adhesion, endoplasmic reticulum, and late endosome (Figure $1 \mathrm{E}$ and Online Supplementary Table S7). Together these clusters included three of the 30 most differentially expressed genes; SNX24, HBE1 and TESPA1. Of these, SNX24 was the most downregulated, having a 45.91-fold reduction in expression in FLI1-deficient platelets compared with normal platelets (false discovery rate $P=0.0034$ ), and was the only one of the nine genes represented in a subgroup of 53 genes from the cluster that was enriched for the GO term 'protein transport' $(P=0.024)$ (Figure $1 \mathrm{E}, \mathrm{F}$ and Online Supplementary Table S8). The 232 genes associated with GO terms in the top four annotation clusters are listed by their gene symbols and descriptions, and expression levels in Online Supplementary Table S9. Further work focused on SNX24. qPCR of SNX24 expression using independent probes confirmed its downregulation in platelet RNA isolated from both P1 and P2 (Online Supplementary Figure S1B).

\section{SNX24 is required during early megakaryopoiesis}

SNX24 is a member of the Sorting nexin family of proteins which are defined by the presence of a Phox homology (PX) phosphoinositide-binding domain and play essential roles in regulating protein trafficking, through all stages of the endocytic pathway. While a specific role for SNX24 in platelets has not been demonstrated, variants of SNX24 have been associated with platelet-crit and mean platelet volume,,$^{27,28}$ and more recently, SNX24 was shown to be upregulated in megakaryocytes with ploidy. ${ }^{29}$ These findings, and our observation that SNX24 was downregulated in FLI1-deficient platelets displaying abnormal $\alpha$-granules, suggest a role for SNX24 in platelet formation which we explored further in iPSC-derived proplatelet-forming megakaryocytes.

We generated an SNX24 KO in iPSC using CRISPR guides targeting the first exon of SNX24 and confirmed the absence of SNX24 expression in two clones by qPCR (Online Supplementary Figure S2A). Further analysis confirmed that both clones were homozygous $\mathrm{KO}$, and that both the wild-type and $\mathrm{KO}$ cells retained normal karyotypes (Online 
A

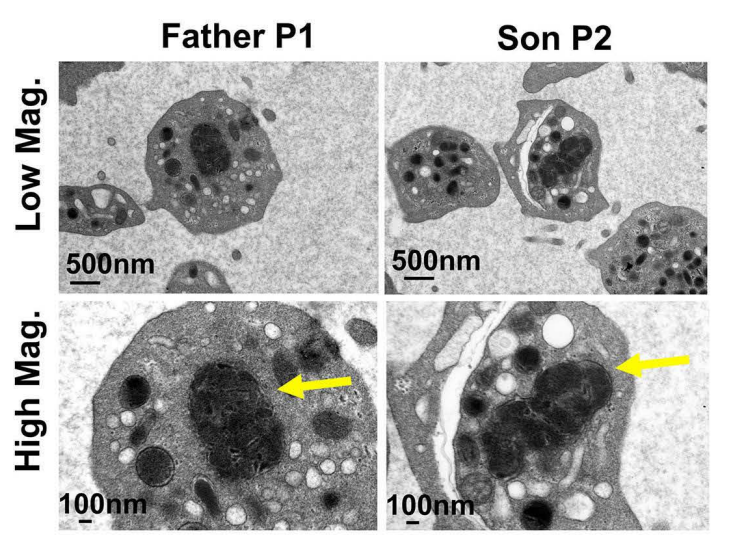

C

E

GO enrichment

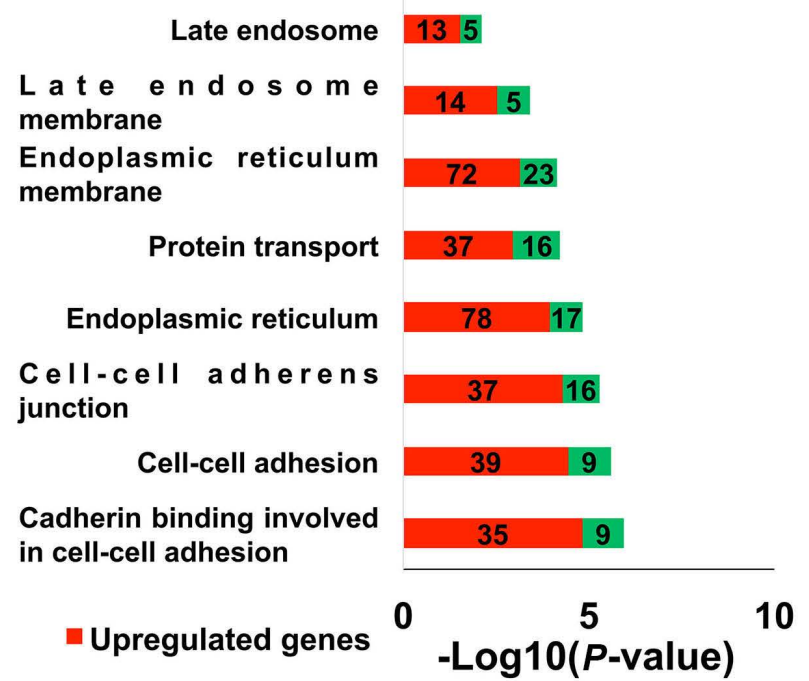

B

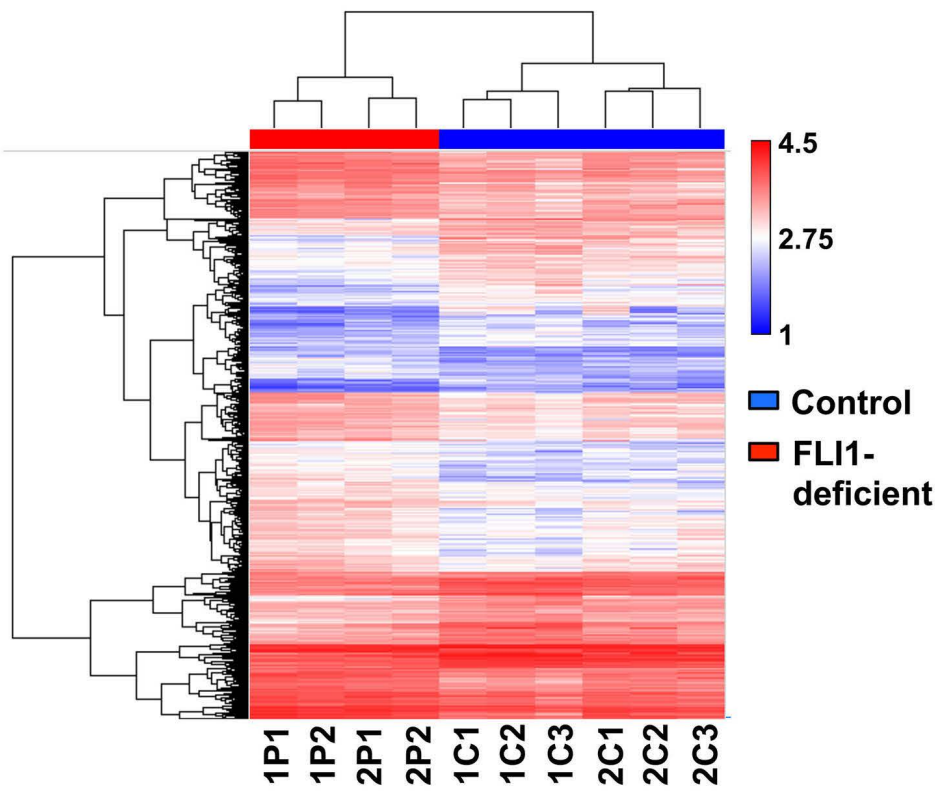

D

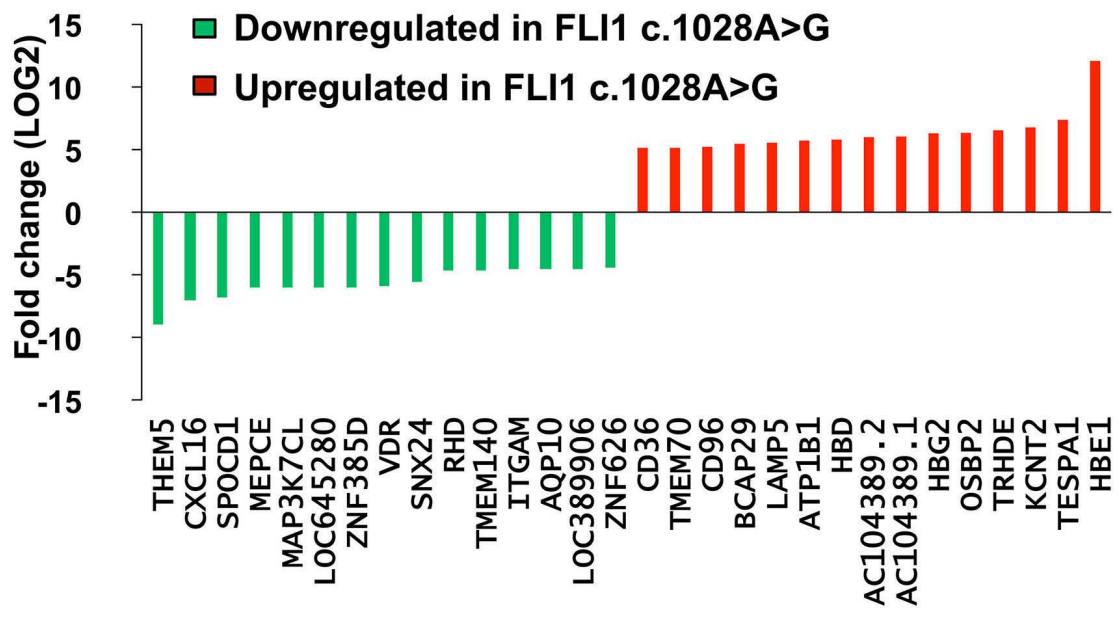

$\mathbf{F}$

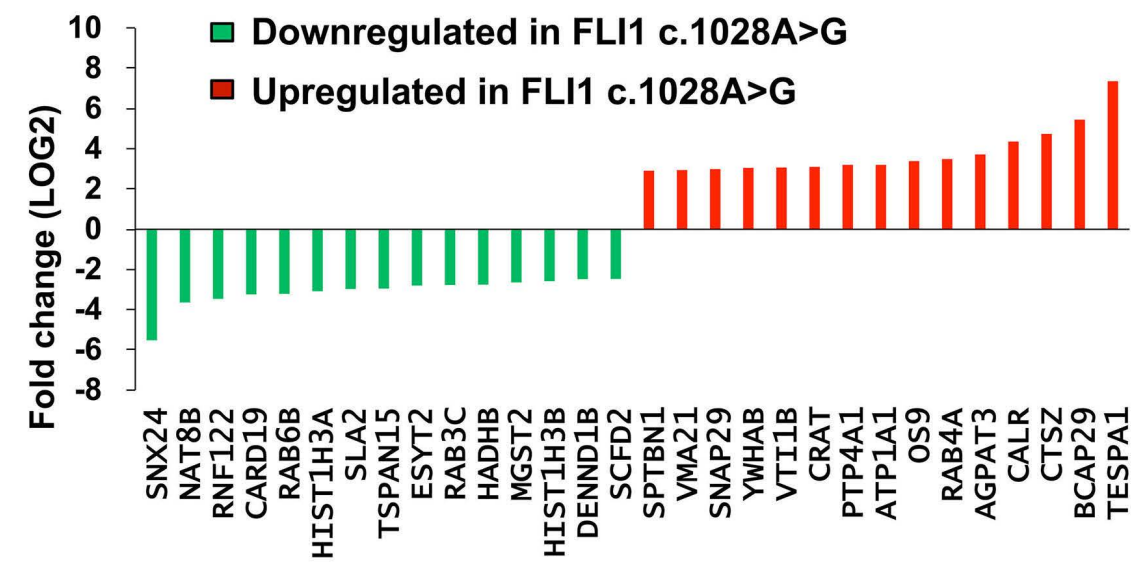

Figure 1. Transcriptome analysis of FLI1-deficient platelets identified SNX24 for further investigation. ( A) Representative electron micrographs of platelets from two subjects, P1 and P2, displaying large and fused -granules (indicated by yellow arrows). (B) Hierarchical clustering of differentially expressed transcripts in platelets from controls (C1, C2, C3) and FLI1-deficient subjects (P1, P2). All samples were analyzed in parallel on two occasions. (C) Volcano plot showing the transcripts that are downregulated (green) or upregulated (red) in FLI1-deficient platelets relative to control platelets. (D) Log fold change of the 30 most downregulated and upregulated coding transcripts observed in FLI1-deficient platelets relative to control platelets $(<2$ or $\geq 2$ fold change, false discovery rate $\leq 0.05$ ). (E) Functional annotation clustering of differentially expressed coding transcripts using DAVID identified 234 annotation clusters. Gene ontology (GO) terms from the top four annotation clusters which were significantly enriched are shown and the $\log _{10} P$-value for each GO term is displayed on the X-axis. The numbers of upregulated and downregulated genes with each GO term are indicated. (F) Log fold change of the 15 most downregulated (green) and upregulated (red) genes showing enrichment for the GO terms protein transport, cell adhesion, endoplasmic reticulum and late endosome (<2 or $\geq 2$ fold change, false discovery rate $\leq 0.05$ ). 
Supplementary Figure $S 2 B-E$ ). We used established procedures to differentiate iPSC $\left(\mathrm{OCT}^{+}\right)$to hematopoietic progenitors derived from hemogenic endothelium $\left(\mathrm{CD} 34^{+}\right.$; day $0-6)$, then to immature megakaryocytes (CD $41^{+} / C D 42^{-}$; day $6-12)$ before terminal differentiation to mature proplatelet-forming megakaryocytes (CD $41^{+} / \mathrm{CD} 42^{+}$; day 12-17) (Figure 2A). qPCR to assess expression of specific markers of stem cells (OCT4), hematopoietic stem cells (CD34) and megakaryocytes (CD61/Gpllla, CD41/GPIIb and $\mathrm{CD} 42 \mathrm{~b} / \mathrm{GPI} \mathrm{b} \alpha$ ) confirmed the expected expression for the different stages of differentiation of the wild-type iPSC (Online Supplementary Figure S3A). Similarly, assessment of FLI1 expression by qPCR showed the expected increase in expression during megakaryocyte maturation as FLI1 regulates the expression of both early and late megakaryocyte-specific genes (Online Supplementary Figure $S 3 B){ }^{2}$ Examination of SNX24 expression during differenti- ation of wild-type iPSC showed that it steadily increased during the early stages of megakaryocyte differentiation and was subsequently downregulated during proplatelet formation (Figure 2B). This suggests that expression of SNX24 is tightly controlled during megakaryopoiesis.

We observed a decrease in expression of the immature megakaryocyte markers (CD41 and CD61) and an increase in expression of the mature megakaryocyte marker (CD42b) at day 12 of differentiation of the SNX24 KO iPSC, suggesting the presence of more mature megakaryocyte progenitors (Figure 2C, D, and Online Supplementary Figure S3C). SNX24 KO iPSC generated megakaryocytes with similar ploidy numbers to the wild-type iPSC, suggesting that depletion of SNX24 does not inhibit polyploidization (Figure 2E). SNX24 KO cells also retained the ability to generate proplatelet-forming megakaryocytes after terminal differentiation (Figure $2 \mathrm{~F}$ ). These data suggest a po-
A

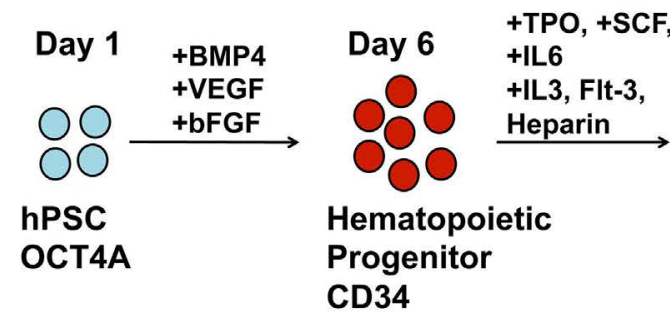

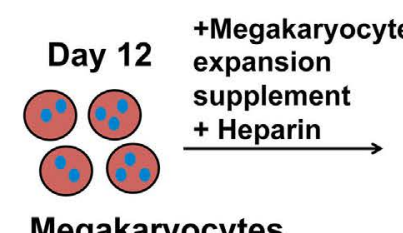

Megakaryocytes CD41/CD42

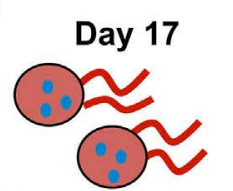

Megakaryocyte Forming Proplatelet CD41/CD42
B

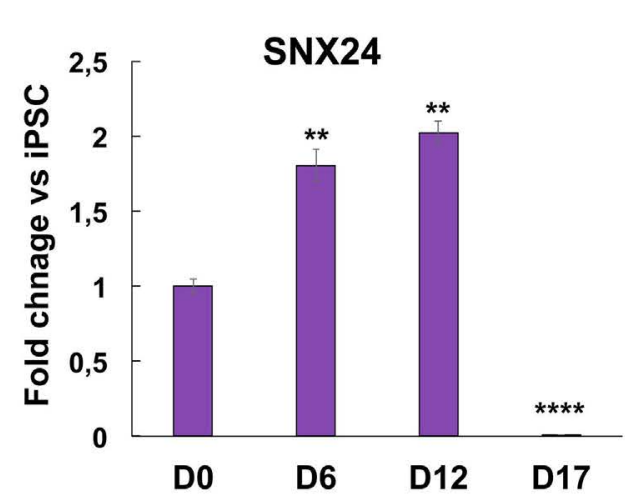

C

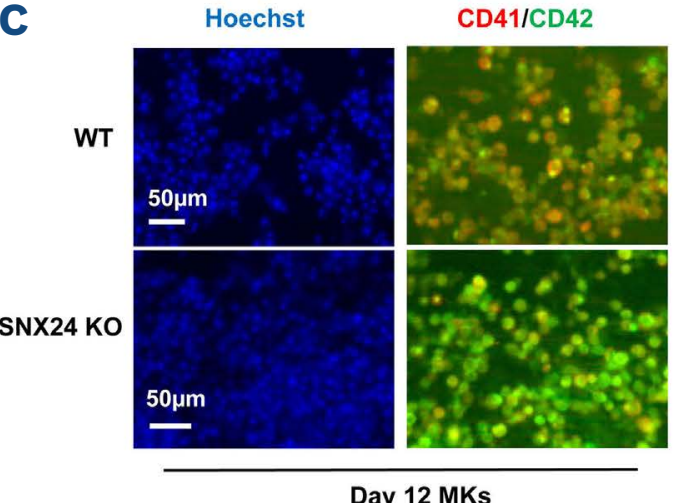

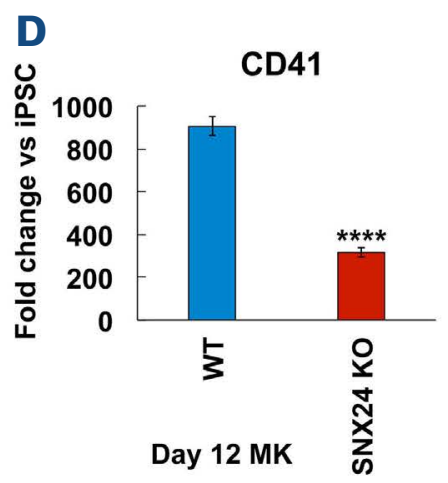

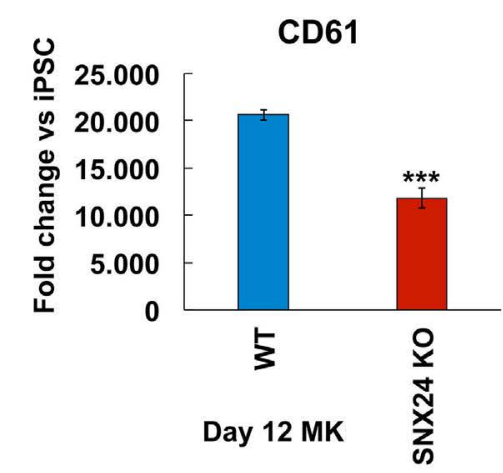

E

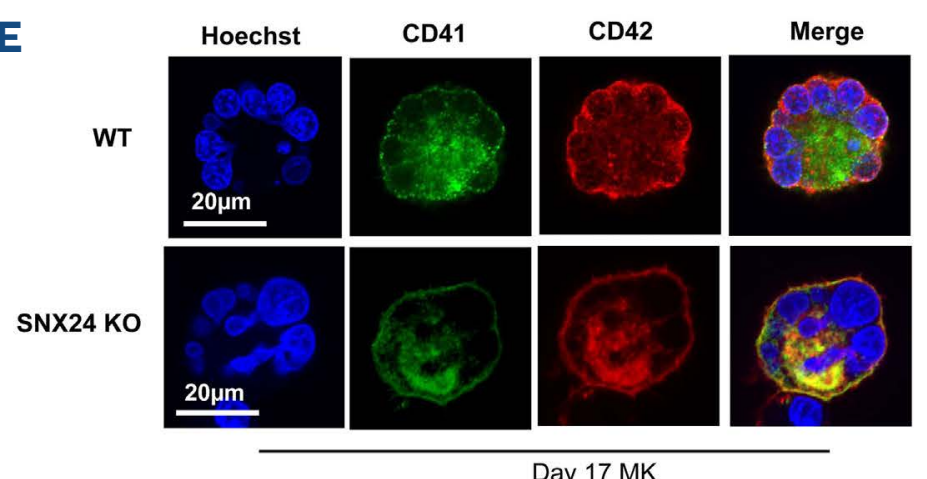

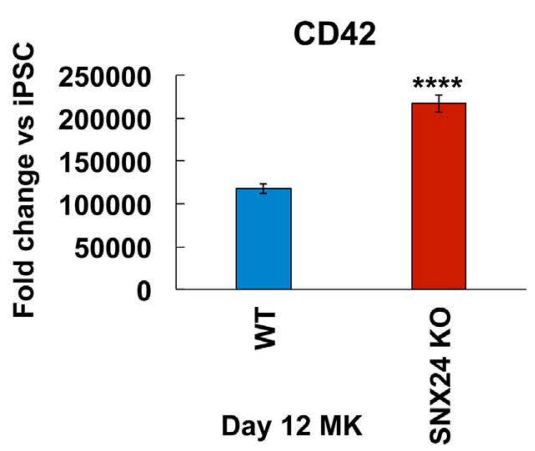

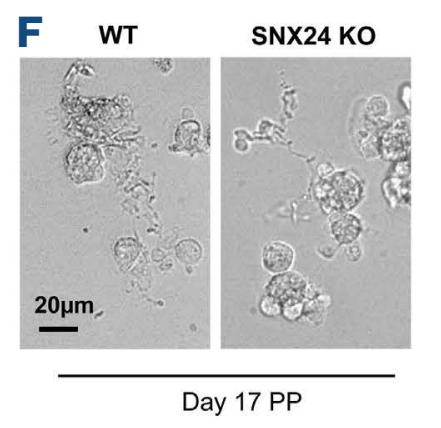

Figure 2. Characterization of megakaryocytes derived from SNX24 knockout induced pluripotent stem cells (A) Schematic of differentiation of induced pluripotent stem cells (iPSC) to megakaryocytes. (B) Quantitative polymerase chain reaction ( $\mathrm{QPCR}$ ) analysis of SNX24 expression during iPSC differentiation to megakaryocytes. $\mathrm{n}=3$ experiments. $* * * * P<0.0001, * * P<0.01$, Student $t$-test. (C) Immunofluorescence staining for CD41 (green) and CD42 (red) in wild-type (WT) cells at day 12 of differentiation. Nuclei are counterstained with Hoechst 33342. Scale bar $50 \mu \mathrm{m}$. (D) qPCR analysis of CD41, CD61 and CD42 gene expression in day 12 WT and SNX24 knockout (KO) megakaryocyte progenitors. $* * * * P<0.0001, * * * P<0.001$. Student $t$-test, $n=2$. (E) Representative confocal microscopy fluorescence images of CD41 (green) and CD42 (red) in WT and SNX24 KO megakaryocytes. Nuclei are counterstained with Hoechst 33342. Scale bar $20 \mu \mathrm{m}$. (F) Brightfield images of proplatelets in WT and SNX24 KO cells. Scale bar $20 \mu \mathrm{m}$. MK: megakaryocytes; PP: proplatelets. 
tential role for SNX24 in the early stages of megakaryopoiesis during megakaryocyte progenitor formation.

\section{SNX24 depletion causes loss of granule content}

To assess whether SNX24 plays a role in granule formation, we examined the ultrastructure of SNX24 KO megakaryocytes by TEM. We observed several organelles in wild-type iPSC-derived megakaryocytes including $\alpha$-granules, $\delta$-granules, endosomal intermediates (multivesicular bodies [MVB] types I and II) and mitochondria. We defined an $\alpha$-granule as a single membrane enclosing a matrix and a $\delta$-granule as a round organelle with a high-density core surrounded by a white rim. There was a dramatic increase in the presence of empty membrane-bound organelles in the SNX24 KO cells, although the mitochondria appeared unaffected (Figure 3A). The empty spherical organelles, multivesicular subclasses and tubular shaped compartments were suggestive of morphologically distinct stages of $\alpha$-granules. $\delta$-granules could not be accurately evaluated in iPSC-derived megakaryocytes, for which unstained electron microscopy would be more informative. Wildtype megakaryocytes contained early endosomal compartments packed with vesicles and intraluminal contents, multivesicular bodies (MVB) with intraluminal vesicles and late endosomes with intraluminal contents. Loss of SNX24 resulted in empty intermediate endosomal compartments during granule biogenesis (Figure 3B). These findings support the participation of SNX24 in the biogenesis and maturation of MVB as well as in the development of $\alpha$-granules.
A
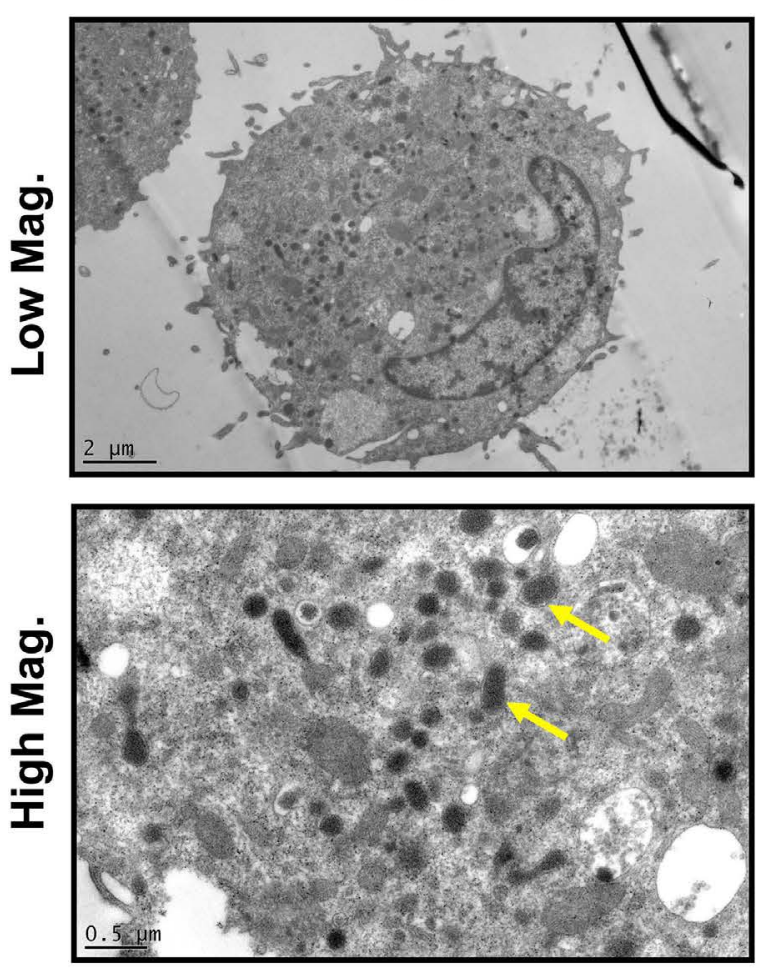

B
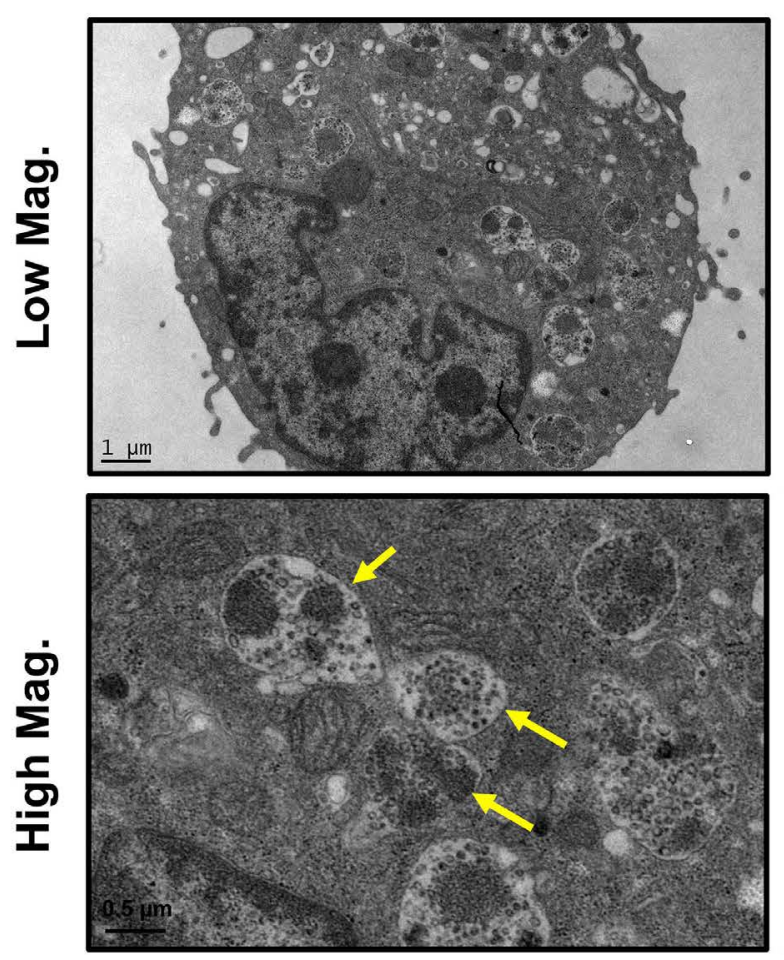

SNX24 KO

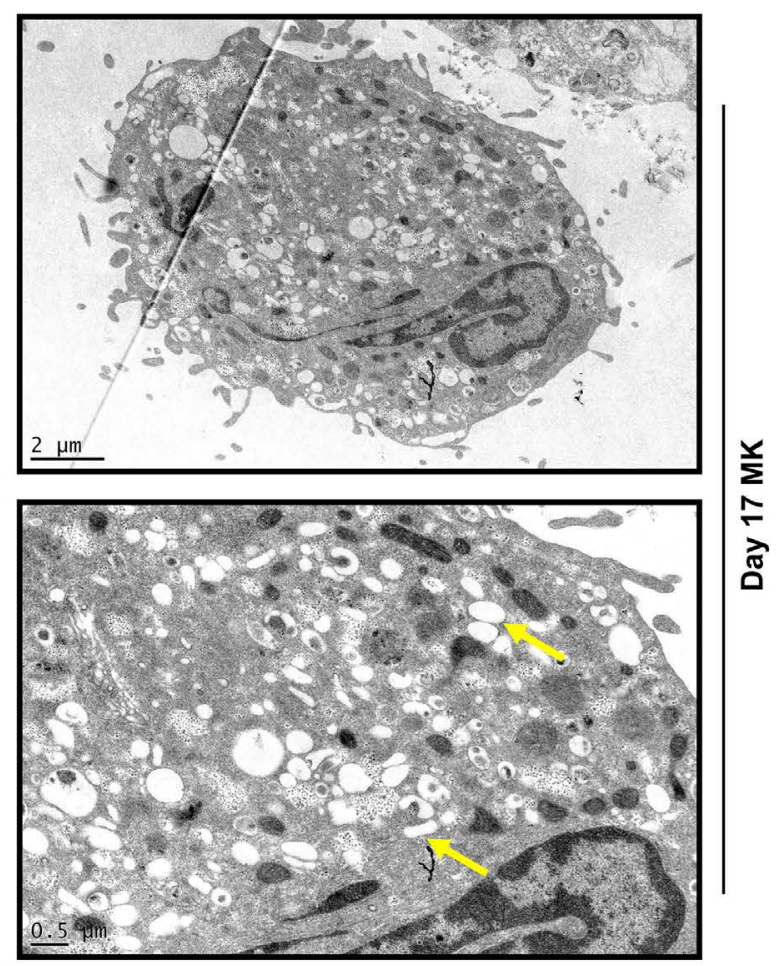

SNX24 KO
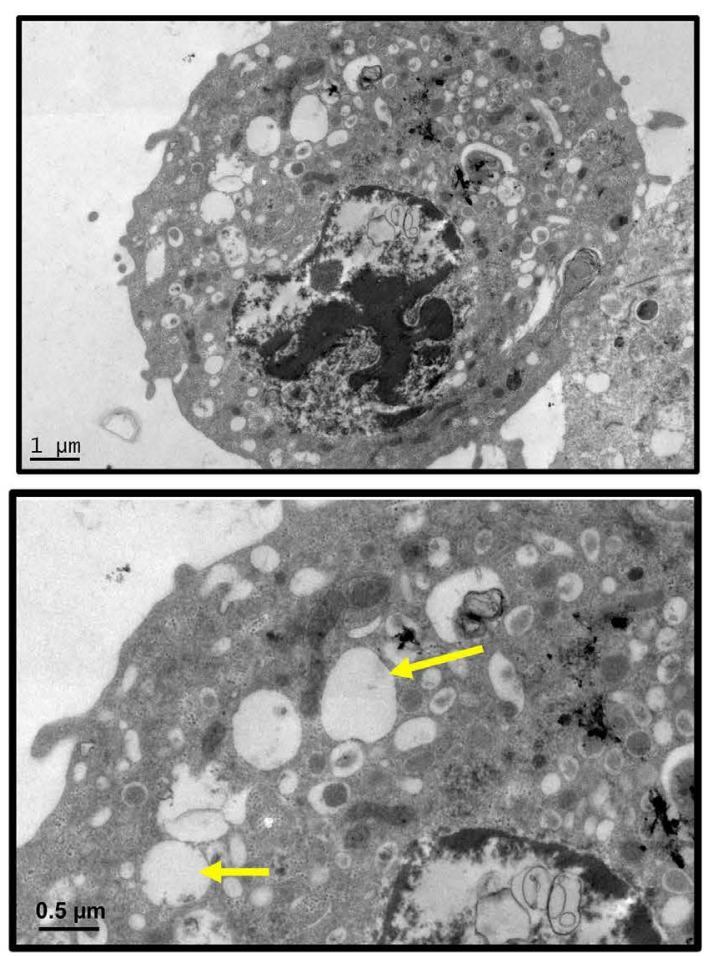

Figure 3. SNX24 knockout megakaryocytes lack $\alpha$-granules (A) Representative transmission electron micrographs of wild-type (WT) and SNX24 knockout (KO) megakaryocytes. Spherical and tubular $\alpha$-granules (arrow heads) are observed in WT cells and absent in SNX24 KO cells. The upper panels are low magnification with a scale bar of $2 \mu \mathrm{m}$. The lower panels are high magnification images with a scale bar of $0.5 \mu \mathrm{m}$. (B) Electron micrographs of WT cells and SNX24 KO cells. Multivesicular bodies (arrow heads) containing intraluminal vesicles were observed in WT cells but not in SNX24 KO cells. The upper panels are low magnification images with a scale bar of $1 \mu \mathrm{m}$. The lower panels are high magnification images with a scale bar of $0.5 \mu \mathrm{m}$. Mag.: magnification; MK: megakaryocytes. 


\section{SNX24 localizes to $\alpha$-granules and endosomal compartments}

We explored whether SNX24 is required for the biogenesis of $\alpha$-granules and endosomal precursors using confocal fluorescence microscopy to examine its co-localization with markers for $\alpha$-granules (CD62P), early endosomes (EEA1), late endosomes and MVB (Rab7a) in megakaryocytes. We observed SNX24 localization in iPSC-derived megakaryocytes, which we identified by multiple nuclei (N). In megakaryocytes, SNX24 partially co-localized with CD62P in punctate structures in $2 \mathrm{~N}$ and $6 \mathrm{~N}$ megakaryocytes (Figure 4A). We observed that SNX24 was localized to both larger $\alpha$-granules and smaller punctate structures, which we speculate could be transport vesicles. SNX24 was also associated with the plasma membrane where it was distributed as small puncta and aggregated clusters, some of which co-localized with CD62P. SNX24 expression was low in the proplatelets but still co-localized to CD62P positive punctate structures (Figure 4B).

SNX24 was partially co-localized with early endosomes in megakaryocytes around the plasma membrane, and associated with EEA1-positive compartments (Figure 4C). SNX24 also associated with Rab7a-positive late endosomes and MVB (Figure 4D). These observations indicate that SNX24 is associated with each stage of $\alpha$-granule maturation, and suggest that it may traffic from early endosomes to mature $\alpha$-granules.
We also examined gene expression of markers specific for early endosomes (Rab5a) and MVB (Rab7a) in SNX24 KO megakaryocytes. Interestingly, Rab5a and Rab7a gene expression was significantly increased in SNX24 KO megakaryocytes compared to the wild-type cells, which was consistent with disruption to the endosomal trafficking pathway (Online Supplementary Figure S4). In contrast, NBEAL2 expression was significantly decreased in the SNX24 KO megakaryocytes, supporting the involvement of SNX24 at an earlier stage of $\alpha$-granule development than NBEAL2 (Online Supplementary Figure S4).

\section{SNX24 knockout cells lack $\alpha$-granule cargo}

The appearance of empty $\alpha$-granules and intermediate endosomal compartments in SNX24 $\mathrm{KO}$ cells is suggestive of a depletion in $\alpha$-granule cargo. Furthermore, the distribution pattern of SNX24 around the cell periphery and in $\alpha$-granule compartments suggests it may be involved in the trafficking of $\alpha$-granule cargo. We therefore examined the subcellular distribution of $\alpha$-granule cargo (CD62P, von Willebrand factor [VWF]) in SNX24 KO megakaryocytes. We observed a reduction in CD62P and VWF staining in megakaryocytes, indicating an $\alpha$-granule trafficking defect (Figure 5A, B). Reverse transcriptase qPCR analysis confirmed that CD62P and VWF gene expression was significantly reduced in SNX24 KO cells (Figure 5C, D). These findings indicate a requirement for SNX24 for traf-
A
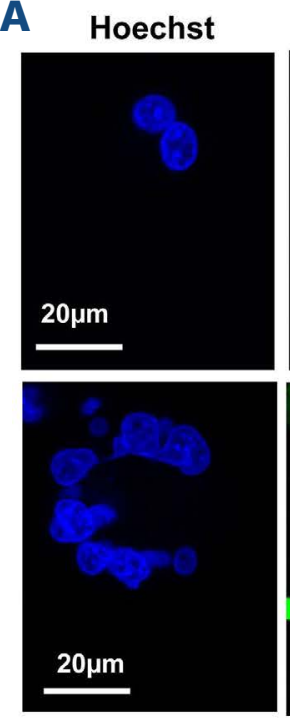

C
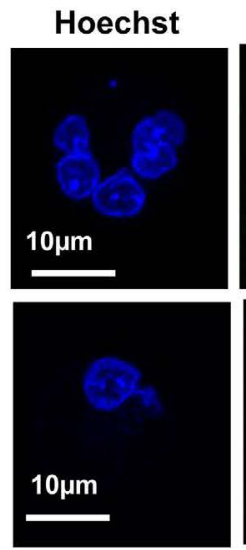
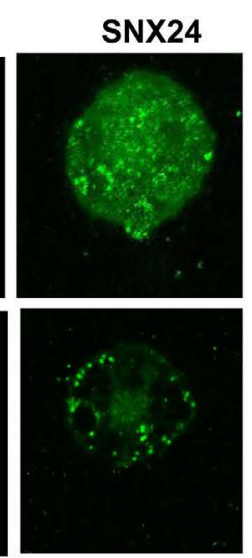

CD62P
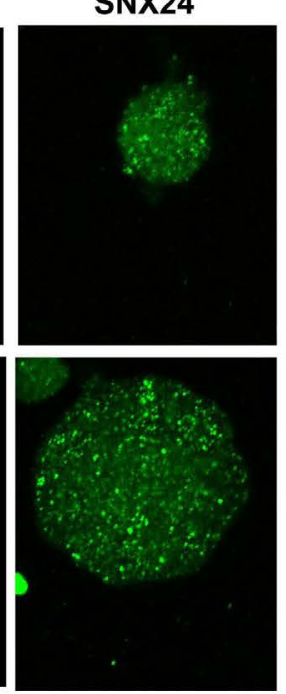
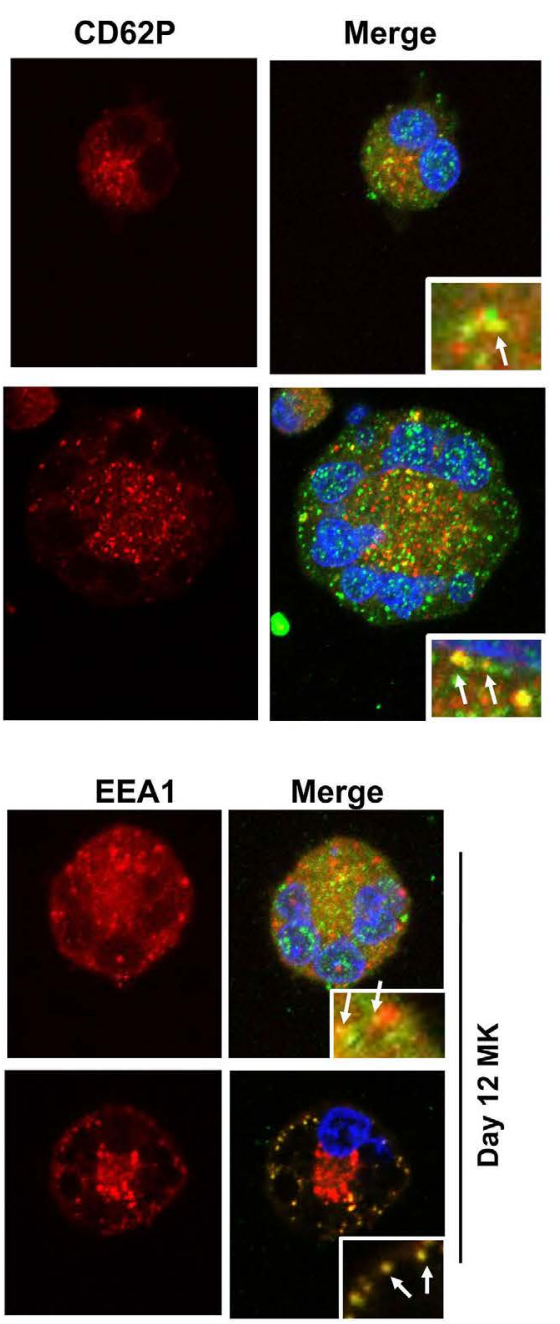

B
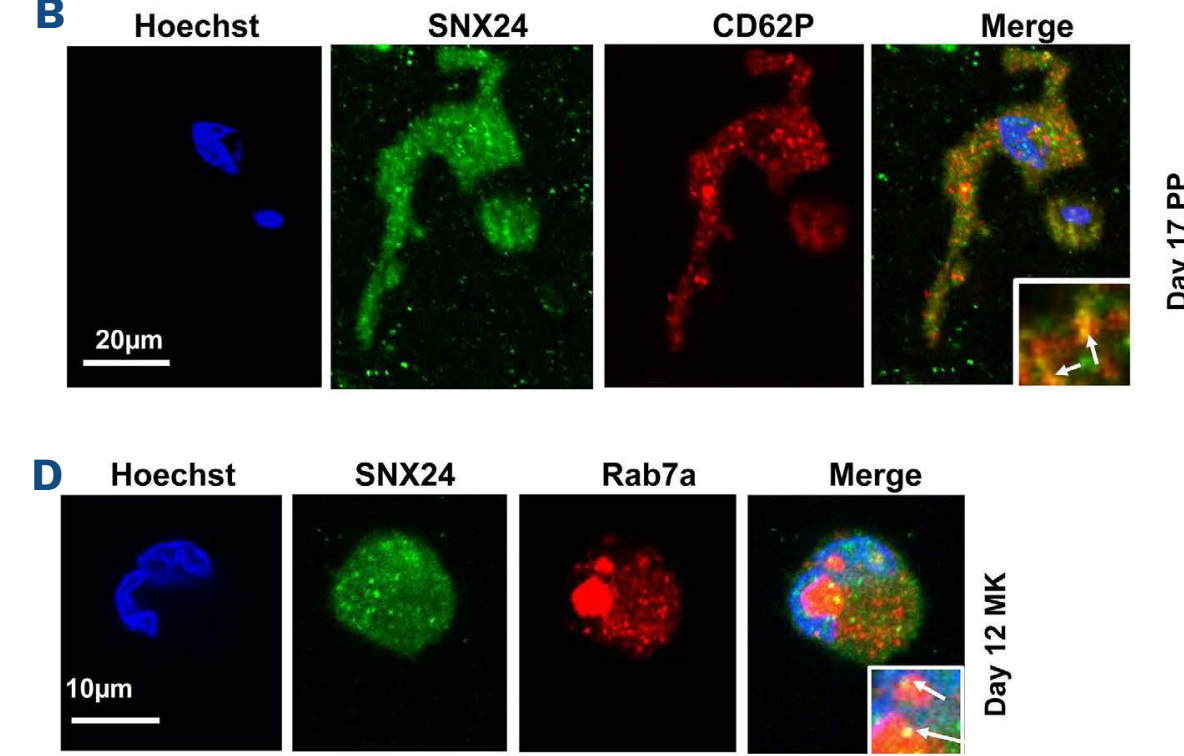

Figure 4. SNX24 $\alpha$ localizes to $\alpha$-granules and endosomal intermediates. (A) Representative confocal microscopy images of wild-type early megakaryocytes (upper panels) and late megakaryocytes (lower panels) stained with anti-SNX24 (green) and anti-CD62 (red). Nuclei are counterstained with Hoechst 33342. Scale bar $20 \mu \mathrm{m}$. (B) Representative confocal microscopy images of wild-type proplatelets stained with anti-SNX24 (green) and anti-CD62 (red). Nuclei are stained with Hoechst 33342. Scale bar $20 \mu \mathrm{m}$. (C) Representative confocal microscopy images of wild-type megakaryocytes stained with anti-SNX24 (green) and anti-EEA1 (red). Nuclei are stained with Hoechst 33342. Scale bar $10 \mu \mathrm{m}$. (D) Representative confocal fluorescence microscopy images of wild-type megakaryocytes immunostained with anti-SNX24 (green) and anti-Rab7a (red). Nuclei are stained with Hoechst 33342. Scale bar $10 \mu \mathrm{m}$. MK: megakaryocytes. 
ficking of both soluble (VWF) and membrane (CD62P) cargo.

\section{Discussion}

The pathways leading to platelet granule biogenesis and exocytosis, and the genes that regulate them, remain to be fully established. In this study, we sought to identify candidate genes that potentially contribute to platelet granule biogenesis by taking advantage of the changes in gene expression that occur in platelets from individuals with germline mutations that affect the transcriptional activity of FLI1, which also display abnormal $\alpha$-granules. Thus, transcriptome analysis of platelets harboring a DNAbinding variant of FLI1 revealed significant differences in expression profiles of FLI1-deficient and wild-type platelets with 2,276 transcripts identified as being differentially expressed in the FLI1-deficient platelets. Functional annotation clustering of the differentially expressed coding transcripts revealed significant enrichment for annotations relating to protein transport, and led us to focus on SNX24, a protein not previously implicated in platelet granule biogenesis. We confirmed that SNX24 expression was significantly downregulated in the FLI1-deficient platelets and observed that SNX24 is localized throughout the pathway of $\alpha$-granule biogenesis, associating with early endosomes, MVB and $\alpha$-granules. Furthermore, loss of SNX24 disrupts the endosomal trafficking pathway in megakaryocytes, resulting in an $\alpha$-granule defect and decreased expression of $\alpha$-granule proteins. Our findings lead us to propose that SNX24 is a novel component of the protein sorting machinery during $\alpha$-granule maturation.

The Sorting nexins (SNX) are a diverse family of cytoplasmic and membrane-associated proteins that are involved in endocytosis, endosomal sorting and endosomal signaling. They are characterized by the presence of a conserved PX domain which binds specific phosphoinositides, facilitating targeting of proteins to distinct endosomal compartments. ${ }^{30}$ Sorting nexins play critical roles in many aspects of cellular function, and dysfunction of SNX proteins has been described in association with a variety of human disorders including neurodegenerative diseases, pathological infection, cancer and cardiovascular disease. ${ }^{31,32}$ SNX24 is one of a subgroup of the SNX protein family that is relatively poorly characterized, although genome-wide association studies have associated variants of SNX24 with platelet-crit and volume. ${ }^{27,28}$ An interesting correlation between the SNX24 single nucleotide polymorphism (rs28891) and complications due to coronary artery aneurysm in Kawasaki disease has also been reported, and in the same study siRNA knockdown of SNX24 expression was shown to significantly decrease expression of the pro-inflammatory cytokines IL-1 $\beta, I L-6$, and IL-8 in lipopolysaccharide-treated human umbilical vein endothelial cells. ${ }^{33}$ Interestingly, $\alpha$-granules contain a wide range of chemokines including IL-8. ${ }^{21}$ Other Sorting nexins have been implicated in platelet granule formation. In particular, SNX17 was identified in a yeast two-hybrid screen as an interaction partner of P-selectin ${ }^{34}$ and it is thought that SNX17 may regulate the endocytosis of P-selectin from the plasma membrane and inhibit trafficking to lysosomes. ${ }^{35}$

The mechanism underlying the significant downregulation of SNX24 in FLI1-deficient platelets is unclear. Previous work which mapped the genome-wide FLI1-binding sites in primary human megakaryocytes indicated that FLI1 does not bind directly to the SNX24 promoter. ${ }^{36}$ The reduced expression is more likely to be an indirect effect of FLI1 loss, potentially through GABPA which acts in concert with FLI1 to regulate megakaryocyte gene expression, since GABPA gene expression was significantly upregulated in the FLI1-deficient platelets and it is predicted to bind to the SNX24 promoter. $^{37}$

We used functional annotation clustering as a tool to prioritize candidate genes encoding proteins that may be involved in platelet granule biogenesis, focusing our attention on the cluster that was enriched for the GO annotation of 'protein transport' which included SNX24. Interestingly, this subgroup also included SEC22B, Homolog $B$ Vesicle Trafficking Protein, a membrane-resident trafficking protein that was recently shown to be required for $\alpha$-granule biogenesis in megakaryocytes and was upregulated in the FLI1-deficient platelets. ${ }^{38}$ Although further work would be required to determine whether any of the remaining genes in this subgroup are required for platelet granule formation, this observation nonetheless supports the use of transcriptome analysis of FLI1-deficient platelets as an approach to identify novel components of the granule biogenesis machinery.

We used iPSC-derived megakaryocytes to study the role of SNX24 during megakaryopoiesis, comparing wild-type cells and SNX24 KO cells which were generated using CRISPR-Cas9 gene editing. SNX24 expression appears to be tightly regulated during megakaryopoiesis, being highest during early megakaryopoiesis at the megakaryocyte progenitor stage, and dropping significantly in mature proplatelet-forming megakaryocytes. We observed that SNX24 KO cells have a higher proportion of mature CD42 $\mathrm{b}^{+}$ megakaryocyte progenitors. However, loss of SNX24 did not inhibit formation of polyploid megakaryocytes or proplatelet-forming megakaryocytes. TEM analysis of SNX24 KO megakaryocytes showed, in contrast to wild-type megakaryocytes, a reduction in $\alpha$-granules and an increase in the presence of empty vacuoles, resembling the phenotype seen in platelets from patients with gray platelet syndrome, which is characterized by the presence 
A

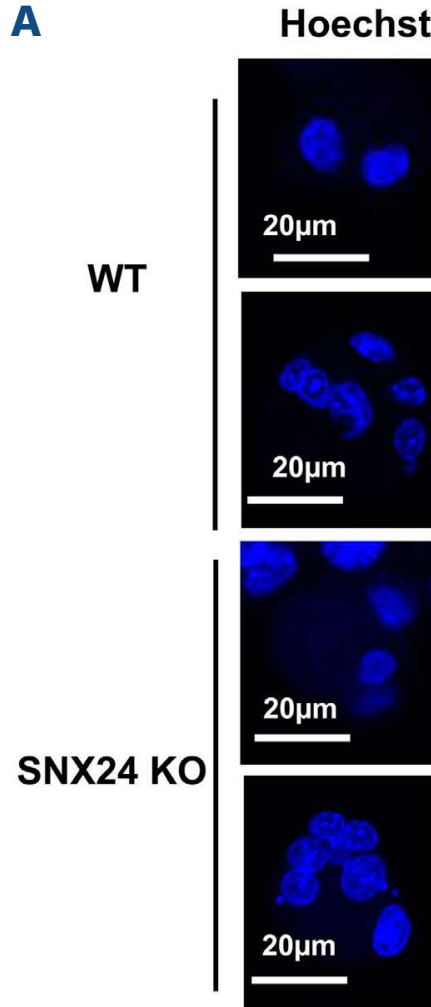

B

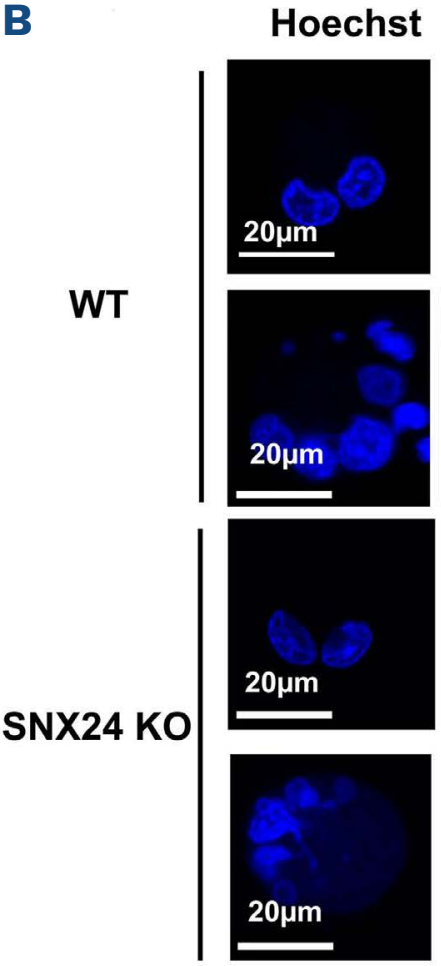

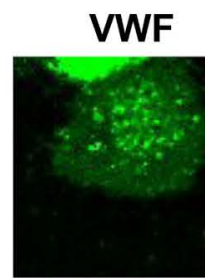
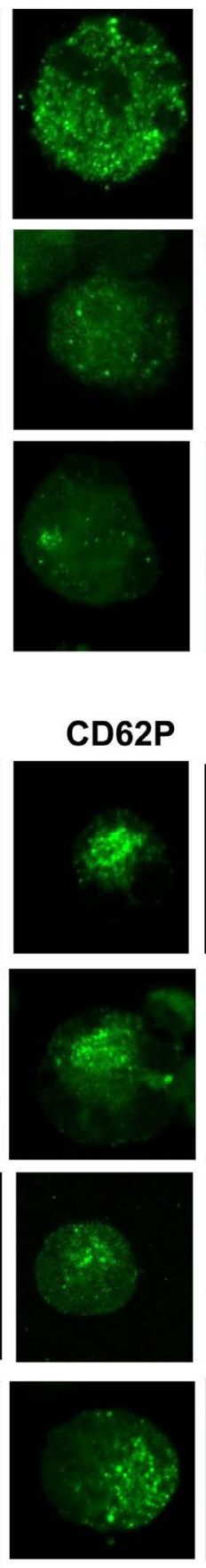

CD42
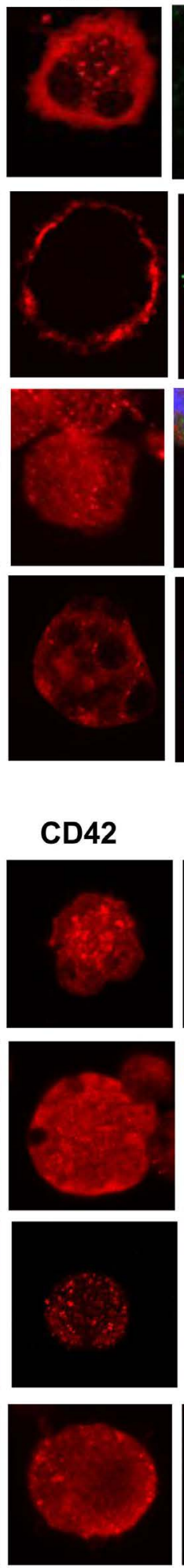
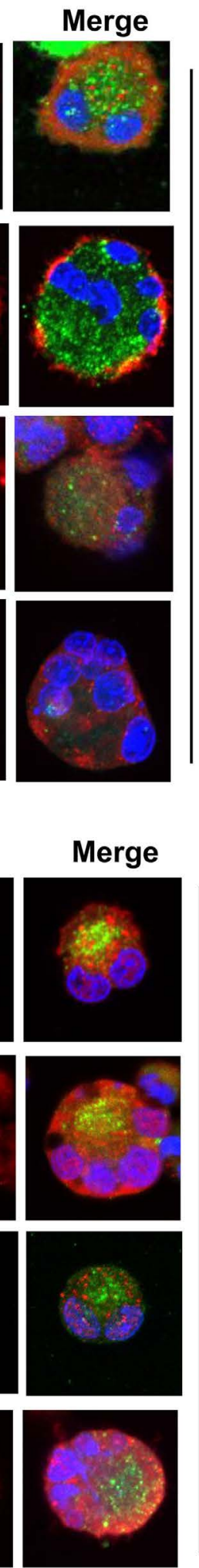

C
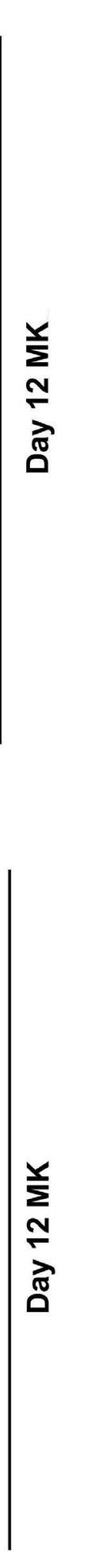

D
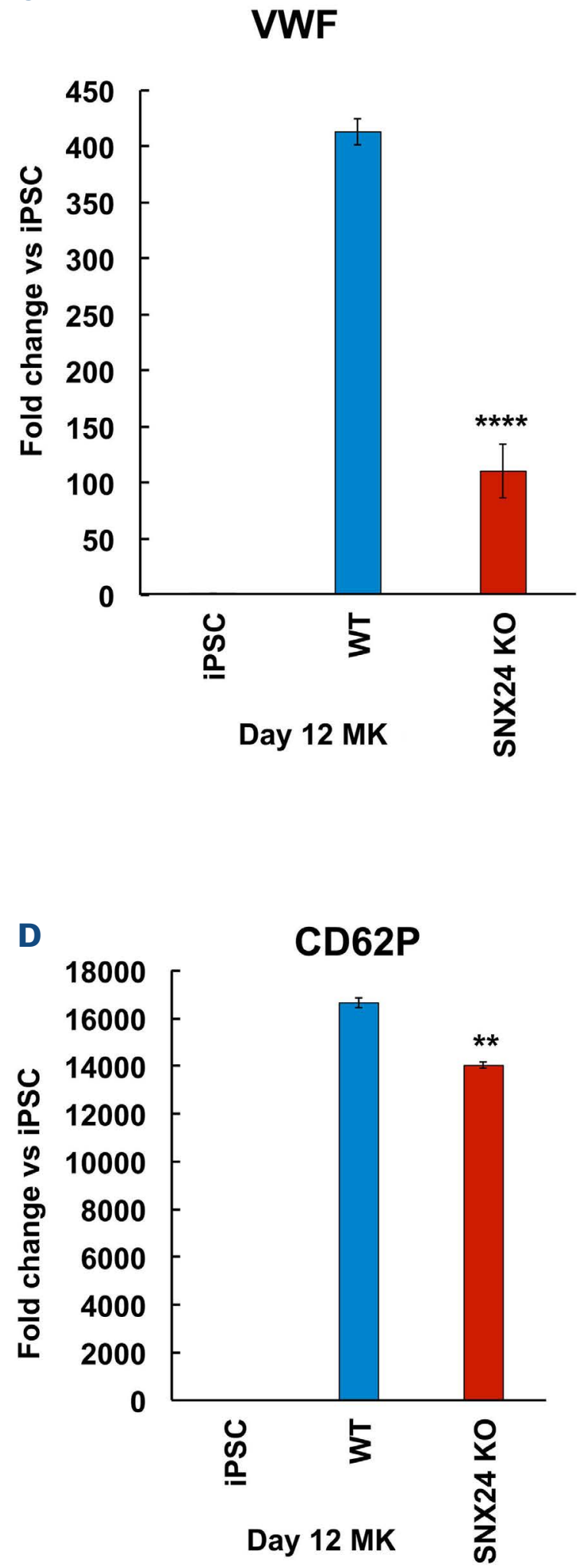

Figure 5. Abnormal trafficking of $\alpha$-granule proteins in SNX24 knockout cells (A) Representative confocal microscopy images of wild-type (WT) and SNX24 knockout (KO) megakaryocytes immunostained with anti-VWF (green) and anti-CD42 (red). Nuclei are stained with Hoechst 33342. Scale bar $20 \mu \mathrm{m}$. (B) Representative confocal microscopy images of WT and SNX24 KO megakaryocytes immunostained with anti-CD62P (green) and anti-CD42 (red). Nuclei are stained with Hoechst 33342 . Scale bar $20 \mu m$. (C) Quantitative polymerase chain reaction (qPCR) analysis of VWF gene expression in day 12 WT and SNX24 KO megakaryocytes. $\star \star \star * P<0.0001$ Student $t$-test, $\mathrm{n}=2$. (D) qPCR analysis of CD62P gene expression in day $12 \mathrm{WT}$ and SNX24 KO megakaryocytes. $\star \star P<0.01$, Student $t$-test, $\mathrm{n}=2$. MK: megakaryocytes; iPSC. Induced pluripotent stem cells.

of empty $\alpha$-granules. ${ }^{39}$ SNX24 KO cells were also devoid of immature type I MVB that contain internal vesicles, and type II MVB that contain internal vesicles and an electron dense core. The ultrastructural abnormalities observed in SNX24 KO megakaryocytes are consistent with defects in the intermediate stages of $\alpha$-granule maturation and resemble those seen in VPS33B KO cells. ${ }^{40}$

Studies in patients with inherited defects that cause deficiencies in $\alpha$-granule cargo and number have yielded valu- able insights into the mechanisms underlying platelet granule biogenesis. Thus, defects in the genes encoding the VPS33B-VPS16B complex, and the BEACH-domain containing NBEAL2, have been shown to underlie the absence of $\alpha$-granules in arthrogryposis, renal dysfunction and cholestasis (ARC) syndrome and in gray platelet syndrome, respectively. ${ }^{18,19,41-43}$ Loss of VPS33B results in the absence of $\alpha$-granules, decreased levels of $\alpha$-granule cargo and a reduction in MVB. ${ }^{40,44}$ Likewise, loss of VPS16B 
results in reduced or undetectable $\alpha$-granule proteins as well as a complete absence of $\alpha$-granules in platelets. ${ }^{41}$ The VPS33B/16B complex localizes on endosomes and promotes protein trafficking between MVB and $\alpha$-granules. ${ }^{45}$ Megakaryocytes deficient in NBEAL2 show a loss of $\alpha$-granules and a reduction in $\alpha$-granule cargo proteins such as VWF but retain some $\mathrm{CD}_{62} \mathrm{P}^{46}$ and Sec22b was recently identified as an interaction partner of NBEAL2, which facilitates $\alpha$-granule cargo stability and granule development. ${ }^{38}$

Our TEM observations indicated an $\alpha$-granule defect in SNX24 KO cells, which led us to assess whether SNX24 was distributed within $\alpha$-granules and intermediate compartments. We have shown that SNX24 is co-localized with $\mathrm{CD} 62 \mathrm{P}$ in $\alpha$-granules during megakaryopoiesis. Vesicles carrying $\alpha$-granule cargo bud off from either the trans-Golgi network or plasma membrane and are subsequently directed to MVB via endosomes. ${ }^{13}$ We show that SNX24 is co-localized with the early endosome marker EEA1 around the cell periphery, and partially associated with Rab7 ${ }^{+}$MVB. This suggests that SNX24 traffics between, or binds to $\alpha$-granule components during their maturation. Likewise, known $\alpha$-granule machinery such as VPS16B and VPS33B traffic between late endosomes and $\alpha$-granules. ${ }^{42}$ We observed that loss of SNX24 leads to a reduction in expression of $\alpha$-granule cargo including VWF and CD62P. P-selectin is a membrane protein that contains a signal peptide to direct it towards a developing granule. ${ }^{47} \mathrm{VWF}$ is a soluble protein that self-assembles into large aggregates and eventually forms tubular structures within $\alpha$-granules. ${ }^{48}$ The differences in expression of VWF and CD62P seen in SNX24 KO cells could reflect differential packaging or vesicle transport to distinct subcompartments within $\alpha$-granules.

Further studies will be required to define the role of SNX24 in intracellular trafficking. In particular, it will be important to investigate its expression throughout megakaryopoiesis and to determine, more precisely, the point at which loss of SNX24 disrupts the trafficking of $\alpha$-granule cargo and leads to the appearance of empty vacuoles, although our findings suggest that this occurs early during maturation as $2 \mathrm{~N}$ megakaryocytes derived from SNX24 KO cells lack $\alpha$-granule cargo. Given its downregulation in mature proplatelet-forming megakaryocytes, and that loss of SNX24 did not inhibit formation of polyploid megakaryocytes or proplatelet-forming megakaryocytes, it would be interesting to determine the role of SNX24, if any, in platelets. There are no disorders reported to be associated with the SNX24 gene, and there are no phenotyping or viability data available for the Snx24 knockout mouse ${ }^{49}$, but it would be interesting to assess the appearance and behavior of their platelets, and to compare them with platelets derived from SNX24 KO megakaryocytes.

In conclusion, we have presented data that evidence a requirement for SNX24 in $\alpha$-granule biogenesis and the intracellular trafficking of $\alpha$-granule cargo within megakaryocytes. Future studies characterizing the molecular interactions of SNX24 will likely reveal further insights into the underlying molecular machinery and protein complexes required for $\alpha$-granule formation in platelets.

\section{Disclosure}

No conflicts of interest to disclose.

\section{Contributions}

$J L$ and SJW designed and performed the experiments; PRH performed the transcriptome analysis; $\mathrm{CJH}$ performed electron microscopy on iPSC-derived megakaryocytes; $L N$ $G$ and BEW performed electron microscopy on clinical samples; MM recruited the patients; AOK provided guidance on the iPSC differentiation; MED and NVM formulated the research idea and designed the study; MED supervised the study; JL analyzed the data, and drafted the first version of the manuscript, which was read and commented on by all authors.

\section{Acknowledgments}

The authors would like to thank the individuals who participated in this study by kindly donating blood samples for transcriptome analysis.

\section{Funding}

This work was supported by the British Heart Foundation through the award of a project grant (PG/15/61/31634). AOK is a Henry Wellcome fellow (218649/Z/19/Z).

\section{Data sharing statement}

Reasonable requests to view the original data will be considered on receipt of an email by Joanne Lacey (j.lacey@sheffield.ac.uk) or Martina Daly (m.daly@sheffield.ac.uk).

\section{References}

1. Li Y, Luo H, Liu T, Zacksenhaus E, Ben-David Y. The ets transcription factor Fli-1 in development, cancer and disease. Oncogene. 2014;34(16):2022-2031.

2. Pang L, Xue H-H, Szalai G, et al. Maturation stage-specific regulation of megakaryopoiesis by pointed-domain Ets proteins.
Blood. 2006;108(7):2198-2206.

3. Hromas R, May W, Denny C, et al. Human FLI-1 localizes to chromosome 11Q24 and has an aberrant transcript in neuroepithelioma. Biochim Biophys Acta. 1993;1172(1-2):155-158. 4. Breton-Gorius J, Favier R, Guichard J, et al. A new congenital 
dysmegakaryopoietic thrombocytopenia (Paris-Trousseau) associated with giant platelet alpha-granules and chromosome 11 deletion at 11q23. Blood. 1995;85(7):1805-1814.

5. Hart A, Melet F, Grossfeld P, et al. Fli-1 is required for murine vascular and megakaryocytic development and is hemizygously deleted in patients with thrombocytopenia. Immunity. 2000;13(2):167-177.

6. Favier R, Jondeau K, Boutard P, et al. Paris-Trousseau syndrome: clinical, hematological, molecular data of ten new cases. Thromb Haemost. 2003;90(5):893-897.

7. Raslova H, Komura E, Le Couédic JP, et al. FLI1 monoallelic expression combined with its hemizygous loss underlies ParisTrousseau/Jacobsen thrombopenia. J Clin Invest. 2004;114(1):77-84.

8. Stockley J, Morgan NV, Bem D, et al. Enrichment of FLI1 and RUNX1 mutations in families with excessive bleeding and platelet dense granule secretion defects. Blood. 2013;122(25):4090-4093.

9. Stevenson WS, Rabbolini DJ, Beutler L, et al. Paris-Trousseau thrombocytopenia is phenocopied by the autosomal recessive inheritance of a DNA-binding domain mutation in FLI1. Blood. 2015;126(17):2027-2030.

10. Saultier P, Vidal L, Canault M, et al. Macrothrombocytopenia and dense granule deficiency associated with FLI1 variants: ultrastructural and pathogenic features. Haematologica. 2017;102(6):1006-1016.

11. Machlus KR, Italiano JE Jr. The incredible journey: from megakaryocyte development to platelet formation. J Cell Biol. 2013;201(6):785-796.

12. Sharda A, Flaumenhaft R. The life cycle of platelet granules. F1000Res. 2018;7:236.

13. Heijnen HF, Debili N, Vainchencker W, Breton-Gorius J, Geuze HJ, Sixma JJ. Multivesicular bodies are an intermediate stage in the formation of platelet alpha-granules. Blood. 1998;91(7):2313-2325.

14. Ambrosio AL, Boyle JA, Di Pietro SM. Mechanism of platelet dense granule biogenesis: study of cargo transport and function of Rab32 and Rab38 in a model system. Blood. 2012;120(19):4072-4081.

15. Chen Y, Yuan Y, Li W. Sorting machineries: how platelet-dense granules differ from $\alpha$-granules. Biosci Rep. 2018;38(5):BSR20180458.

16. Behnke O. Coated pits and vesicles transfer plasma components to platelet granules. Thromb Haemost. 1989;62(2):718-722.

17. Wei AH, Li W. Hermansky-Pudlak syndrome: pigmentary and non-pigmentary defects and their pathogenesis. Pigment Cell Melanoma Res. 2013;26(2):176-192.

18. Gunay-Aygun M, Falik-Zaccai TC, Vilboux T, et al. NBEAL2 is mutated in gray platelet syndrome and is required for biogenesis of platelet $\alpha$-granules. Nat Genet. 2011;43(8):732-734.

19. Gissen P, Johnson CA, Morgan NV, et al. Mutations in VPS33B, encoding a regulator of SNARE-dependent membrane fusion, cause arthrogryposis-renal dysfunction-cholestasis (ARC) syndrome. Nat Genet. 2004;36(4):400-404.

20. Kahr WH, Lo RW, Li L, et al. Abnormal megakaryocyte development and platelet function in Nbeal2(-/-) mice. Blood. 2013;122(19):3349-3358.

21. Blair P, Flaumenhaft R. Platelet alpha-granules: basic biology and clinical correlates. Blood Rev. 2009;23(4):177-189.

22. Nurden P, Stritt S, Favier R, Nurden AT. Inherited platelet diseases with normal platelet count: phenotypes, genotypes and diagnostic strategy. Haematologica. 2021;106(2):337-350.
23. Huang DW, Sherman BT, Lempicki RA. Systematic and integrative analysis of large gene lists using DAVID bioinformatics resources. Nat. Protoc. 2009;4(1):44-57.

24. Huang DW, Sherman BT, Lempicki RA. Bioinformatics enrichment tools: paths toward the comprehensive functional analysis of large gene lists. Nucleic Acids Res. 2009;37(1):1-13.

25. Feng Q, Shabrani N, Thon JN, et al. Scalable generation of universal platelets from human induced pluripotent stem cells. Stem Cell Reports. 2014;3(5):817-831.

26. Khan AO, Slater A, Maclachlan A, et al. Post-translational polymodification of $\beta 1$-tubulin regulates motor protein localisation in platelet production and function. Haematologica. 2022;107(1):243-259.

27. Astle WJ, Elding $\mathrm{H}$, Jiang $\mathrm{T}$, et al. The allelic landscape of human blood cell trait variation and links to common complex disease. Cell. 2016;167(5):1415-1429.

28. Vuckovic D, Bao EL, Akbari P, et al. The polygenic and monogenic basis of blood traits and diseases. Cell. 2020;182(5):1214-1231.

29. Choudry FA, Bagger FO, Macaulay IC, et al. Transcriptional characterization of human megakaryocyte polyploidization and lineage commitment. J Thromb Haemost. 2021;19(5):1236-1249.

30. Haft CR, de la Luz Sierra M, Barr VA, Haft DH, Taylor SI. Identification of a family of sorting nexin molecules and characterization of their association with receptors. Mol Cell Biol. 1998;18(12):7278-7287.

31. Hanley SE, Cooper KF. Sorting nexins in protein homeostasis. Cells. 2020;10(1):17.

32. Yang J, Villar VAM, Rozyyev S, Jose PA, Zeng C. The emerging role of sorting nexins in cardiovascular diseases. Clin Sci (Lond). 2019;133(5):723-737.

33. Lin YJ, Chang JS, Liu X, et al. Sorting nexin 24 genetic variation associates with coronary artery aneurysm severity in Kawasaki disease patients. Cell Biosci. 2013;3(1):44.

34. Florian V, Schlüter T, Bohnensack R. A new member of the sorting nexin family interacts with the $\mathrm{C}$-terminus of P-selectin. Biochem Biophys Res Commun. 2001;281(4):1045-1050.

35. Williams R, Schlüter T, Roberts MS, Knauth $\mathrm{P}$, Bohnensack R, Cutler DF. Sorting nexin 17 accelerates internalization yet retards degradation of P-selectin. Mol Biol Cell. 2004;15(7):3095-3105.

36. Tijssen MR, Cvejic A, Joshi A, et al. Genome-wide analysis of simultaneous GATA1/2, RUNX1, FLI1, and SCL binding in megakaryocytes identifies hematopoietic regulators. Dev Cell. 2011;20(5):597-609.

37. Fishilevich S, Nudel R, Rappaport N, et al. GeneHancer:genomewide integration of enhancers and target genes in GeneCards. Database (Oxford). 2017;2017:bax028.

38. Lo RW, Li L, Pluthero FG, Leung R, Eto K, Kahr WHA. The endoplasmic reticulum protein SEC22B interacts with NBEAL2 and is required for megakaryocyte $\alpha$-granule biogenesis. Blood. 2020;136(6):715-725.

39. Maynard DM, Heijnen HF, Gahl WA, Gunay-Aygun M. The $\alpha-$ granule proteome: novel proteins in normal and ghost granules in gray platelet syndrome. J Thromb Haemost. 2010;8(8):1786-1796.

40. Bem D, Smith H, Banushi B, et al. VPS33B regulates protein sorting into and maturation of $\alpha$-granule progenitor organelles in mouse megakaryocytes. Blood. 2015;126(2):133-143.

41. Urban D, Li L, Christensen $\mathrm{H}$, et al. The VPS33B-binding protein VPS16B is required in megakaryocyte and platelet $\alpha$-granule biogenesis. Blood. 2012;120(25):5032-5040.

42. Kahr WH, Hinckley J, Li L, et al. Mutations in NBEAL2, encoding a $\mathrm{BEACH}$ protein, cause gray platelet syndrome. Nat Genet. 
2011;43(8):738-740.

43. Albers CA, Cvejic A, Favier R, et al. Exome sequencing identifies NBEAL2 as the causative gene for gray platelet syndrome. Nat Genet. 2011;43(8):735-737.

44. Lo B, Li L, Gissen P, Christensen H, et al. Requirement of VPS33B, a member of the Sec1/Munc18 protein family, in megakaryocyte and platelet alpha-granule biogenesis. Blood. 2005;106(13):4159-4166.

45. Ambrosio AL, Di Pietro SM. Mechanism of platelet $\alpha$-granule biogenesis: study of cargo transport and the VPS33B-VPS16B complex in a model system. Blood Adv. 2019;3(17):2617-2626.

46. Lo RW, Li L, Leung R, Pluthero FG, Kahr WHA. NBEAL2

(NeurobeachinLike 2) Is required for retention of cargo proteins by $\alpha$-granules during their production by megakaryocytes. Arterioscler Thromb Vasc Biol. 2018;38(10):2435-2447.

47. Disdier M, Morrissey JH, Fugate RD, Bainton DF, McEver RP. Cytoplasmic domain of P-selectin (CD62) contains the signal for sorting into the regulated secretory pathway. Mol Biol Cell. 1992;3(3):309-321.

48. Huang RH, Wang Y, Roth R, et al. Assembly of Weibel-Palade body-like tubules from $\mathrm{N}$-terminal domains of von Willebrand factor. Proc Natl Acad Sci U S A. 2008;105(2):482-487.

49. Dickinson, M, Flenniken A, Ji X, et al. High-throughput discovery of novel developmental phenotypes. Nature. 2016;537(7621):508-514. 